\title{
BELGIAN CONGO
}

I arrived at Aba on May 3rd in company with Mr. Edgar of Toc $\mathrm{H}$. We were met by Mr. Harrison, of the Heart of Africa Mission, who had kindly offered to provide transport. We inspected the small leper camp at Aba with Dr. Kleinschmidt. With one possible exception the 30 patients were of the neural type, with tuberculoid or simple macules and secondary deformities of hands and feet. Some appeared to be very anaemic and to be suffering from septic and other skin complications. General treatment and treatment of complicating diseases should improve these patients considerably, but due to lack of staff these have not been available. From the point of view of the control of leprosy in the district, such a camp appears to be of little value, as the cases segregated were apparently not of an infectious type.

From Aba we went, via Niangara, to Ibambi, the headquarters of the Heart of Africa Mission, where we were the guests of Mr. and Mrs. Harrison. 'On the way we visited two Chiefs' Leper Camps.

At the first of these, near Dingba, we saw 55 patients, of which 7 appeared to be lepromatous. Of the remaining 48 neural cases, 7 had distinct major or minor tuberculoid lesions. The rest showed secondary neural lesions and deformities, and many of them had simple macules. The patients were tended by a native dresser and visited occasionally by a doctor and a Catholic mission sister.

At the second camp we saw 76 patients, though we were informed that there were more on the other side of the river. Of the 76 cases, 4 appeared to be of the lepromatous type, and of the remaining 72 neural cases ${ }_{5} 5$ showed distinctly tuberculoid lesions; the great majority had ntarked secondary neural deformities. Many of them were obviously anacmic and suffering from complicating skin disorders. Little appeared to be done for their treatment and we were informed that they maintained themselves by their own agriculture.

\section{Pawa Rresarch Station}

After two days stay at Ibambi we went to Pawa, the leprosy research station of the Belgian Croix Rouge. There we were hospitably received by Prof. Dubois and Dr. and Mrs. de Gotte. From the 7 th till the IIth of May I had the privilege of making an intensive study of leprous patients under Prof. Dubois' expert guidance. Besides studying the patients in residence at the Pawa Centre we also visited two leper camps in the neighbourhood, at Ata Kobo and Bengwe. 

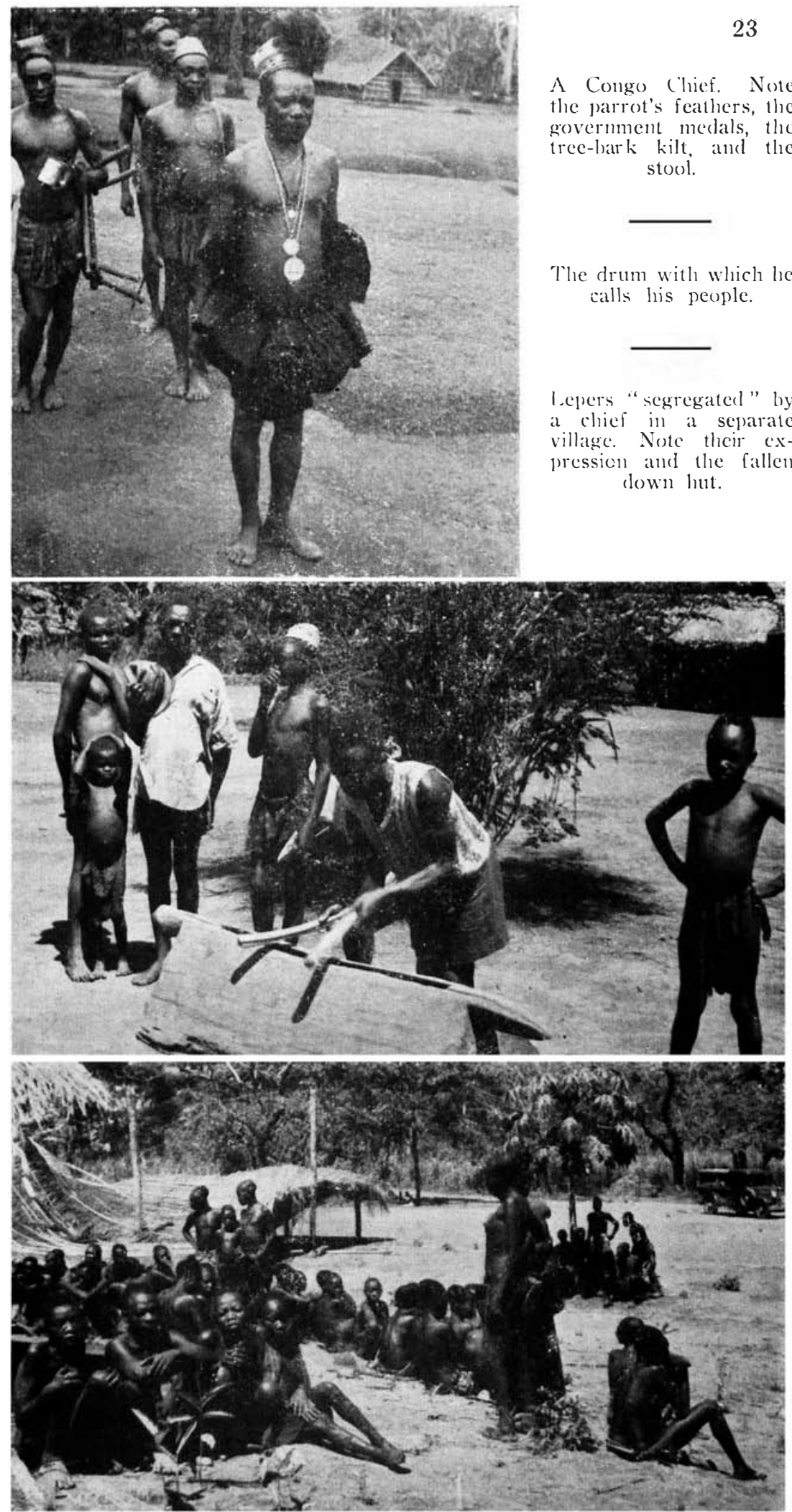
I had already visited Ata Kobo with Mr. Harrison but had an opportunity of studying the patients in detail on this second occasion. Of 242 patients examined, 24 or Io $\%$, were distinctly of the lepromatous type $\left(\mathrm{L}_{2}\right.$ and $\left.\mathrm{L}_{3}\right)$, the rest being chiefly neural cases with marked secondary neural lesions. Sixty seven $(27.7 \%)$ showed distinct tuberculoid lesions. There were also a few with diffuse lepromatous lesions (LD-I) with neither macules nor nodules.

At Bengwe, which we visited on May 9th, the types were very similar in nature and proportions to those at Ata Kobo, only that there were fewer with distinct tuberculoid lesions. This is probably due to the fact that the patients at Bengwe are under regular treatment, while at Ata Kobo no treatment is given.

At these Chiefs' camps one has an excellent opportunity of studying a cross section of the leprous population in each Chief's area as, at the orders of Government, each Chief segregates all cases of leprosy in his area. Doubtless some cases escape detection; but there are not likely to be many, as the Chiefs are expert at recognising the disease. In the Stanleyville Province (one of the six provinces of Congo Belge) there were $7 \mathrm{I}$ of these villages ayricoles organised in I936, with 79I4 lepers.

One of the most valuable methods of rescarch at the Pawa Centre is the repeated census examination of the whole population. At the first examination, which was carried out by an agent sanitaire, and not by an expert doctor, it was calculated that about ten per cent of the population was suffering from leprosy. However, later examination by Prof. Dubois and his colleagues showed that many with non-leprous marks mistaken for leprosy had been included, and that the actual number was round about four per ceint., or, including slight, abortive cases, six or seven per cent. It was found that many of the distinct, but slight cases, were not progressive, their lesions remaining stationary over periods of years in some cases, and others clearing up spontaneously. Also cases which a year before had shown no recognisable signs appeared with wide-spread macular lesions, developing into lepromatous cases.

Central African Leprosy Compared with timat of N. India

One of the most interesting features of the study of leprosy at Pawa, and also in the Equatorial Province of the Sudan, where the disease is very similar, is the marked differences noted between the types of leprosy there and those in North India. These are enumerated below:

(a) Absence of " lepra reaction " and of signs of sensitization to M. leprae. Out of the many hundred cases I examined in 
Congo Belge and S. Sudan I found only one single case of this condition, which is comparatively common in $\mathrm{N}$. India.

(b) The comparative infrequency of $\mathrm{L}_{2}$ and $\mathrm{L}_{3}$ cases. These were found to be about Io per cent, compared with about 20 per cent or more in $\mathrm{N}$. India. Also the proportion of lepromatous, as compared with neural, macules appears to be less than in $\mathbf{N}$. India.

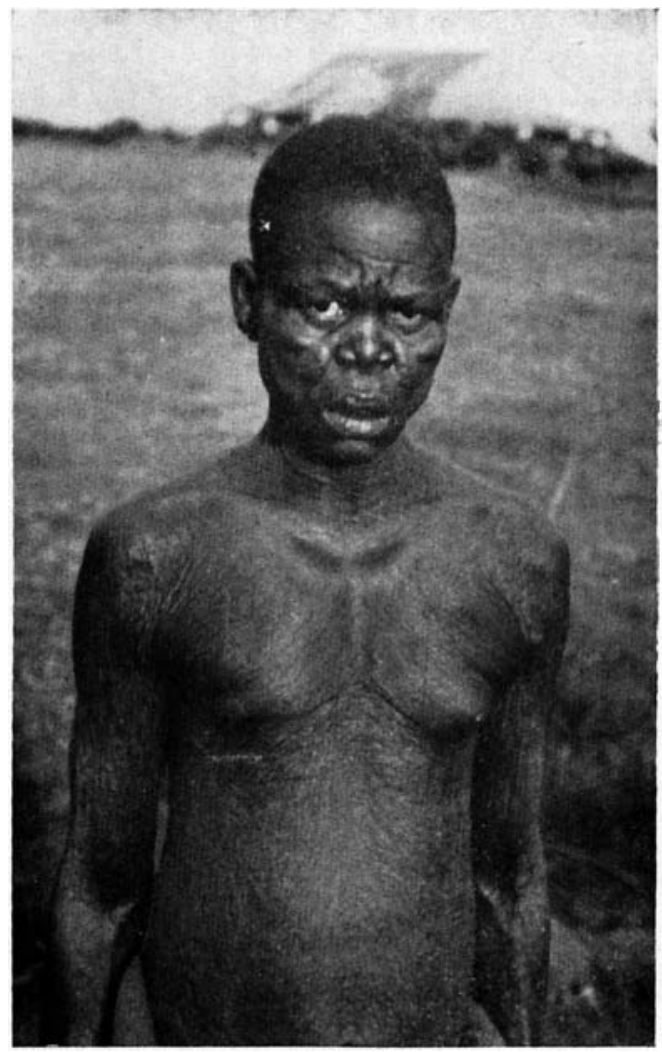

This man is the only case of lepra reaction seen out of many hundreds of patients in the Belgian Congo.

(c) Among the neural cases there appears to be a larger proportion with deformities of the hands and feet than in $\mathrm{N}$. India. This may be the result of infection with jiggers complicated by septic infection.

(d) The deformities of the hand consist of shortening of the digits, but the wasting of the small muscles of the hand followed by main-en-griffe (claw hand) is seldom seen, or only in a mild degree; whereas in $\mathbf{N}$. India these latter signs are as a rule present. 
(c) In the feet the digits are similarly shortened, or have entirely disappeared, to a greater degree than in N. India; but, while trophic ulcers are present, these are comparatively superficial and slight compared with the deep perforating ulcers down to and involving the bone, which is such a common condition in $\mathrm{N}$. India.

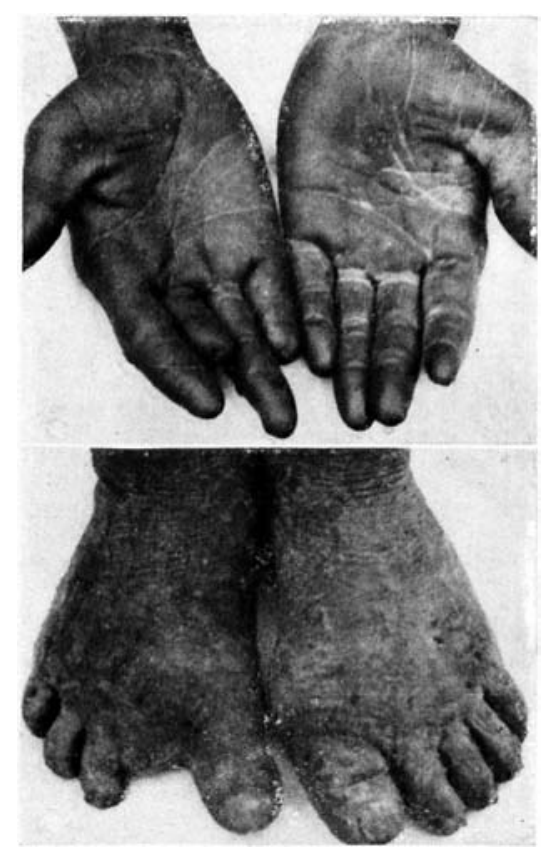

A shortening of fingers and toes in neural leprosy with little wasting of small muscles or deformity, probahly caused lyy jiggers in anaesthetic fingers, followed by sepsis.

(f) The number of tuberculoid cases is perhaps almost as high as in $\mathrm{N}$. India, but in these the presence of anaesthesia to light touch is much less marked than in the latter country. In confirming the diagnosis it is therefore necessary to rely more upon other signs such as analgesia on pricking with a pin, or anhydrosis.

(g) Thickening and tenderness of nerves is much less frequent and, when present, less marked than in N. India. I did not find a single case of nerve abscess (so common in $\mathrm{N}$. India) among the many hundred cases I examined in Congo Belge and the S. Sudan. It is significant however, that I found one nerve abscess among the sixteen cases examined in the N. Sudan (p. I4).

(h) The proportion of neural to lepromatous macules is 
greater than in $\mathbf{N}$. India, simple neural macules being largely responsible for this larger proportion.

Lack of time and opportunity has made it impossible to support these interesting contrasts in type of leprosy with statistical evidence, and the whole question of differences in type in different

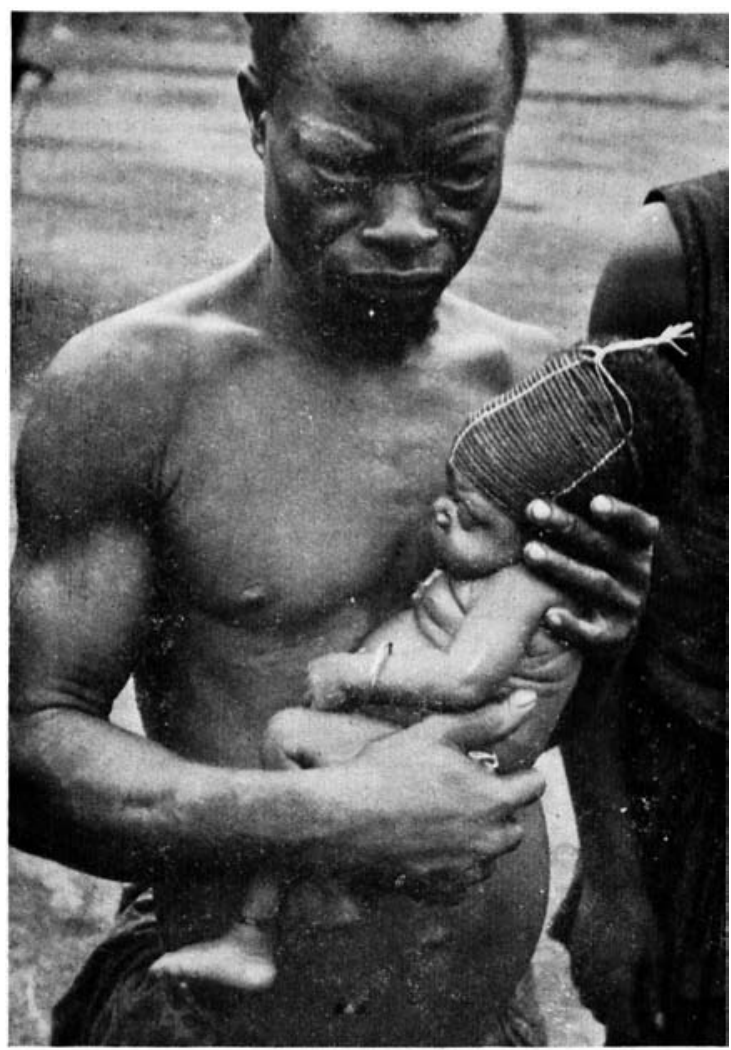

(ongio Fasmlons: Head-lindling which gives an elongated head, does not interfere with the development of the brain, but as it also elongates the eye it may affect vision.

races, climates, and social, dietetic and economic conditions calls for careful and expert study.

It seems clear, however, that leprosy in the N.E. Belgian Congo and the adjoining S. Sudan is of a milder type than in $\mathrm{N}$. India. This is evidenced by the smaller proportion of the more severe lepromatous type, and the absence, or at least extreme rarity, of "lepra reaction". This mildness is also shown by less swelling and tenderness of the nerves, with consequent less anaesthesia to 
light touch and comparative exemption of the small muscles of the hand and the less deep trophic ulcers of the feet.

The question arises as to whether M. leprae as found in Congo Belge and S. Sudan is of a less virulent type than that in N. India. Until a satisfactory experimental animal is available it will be difficult or impossible to answer this question.

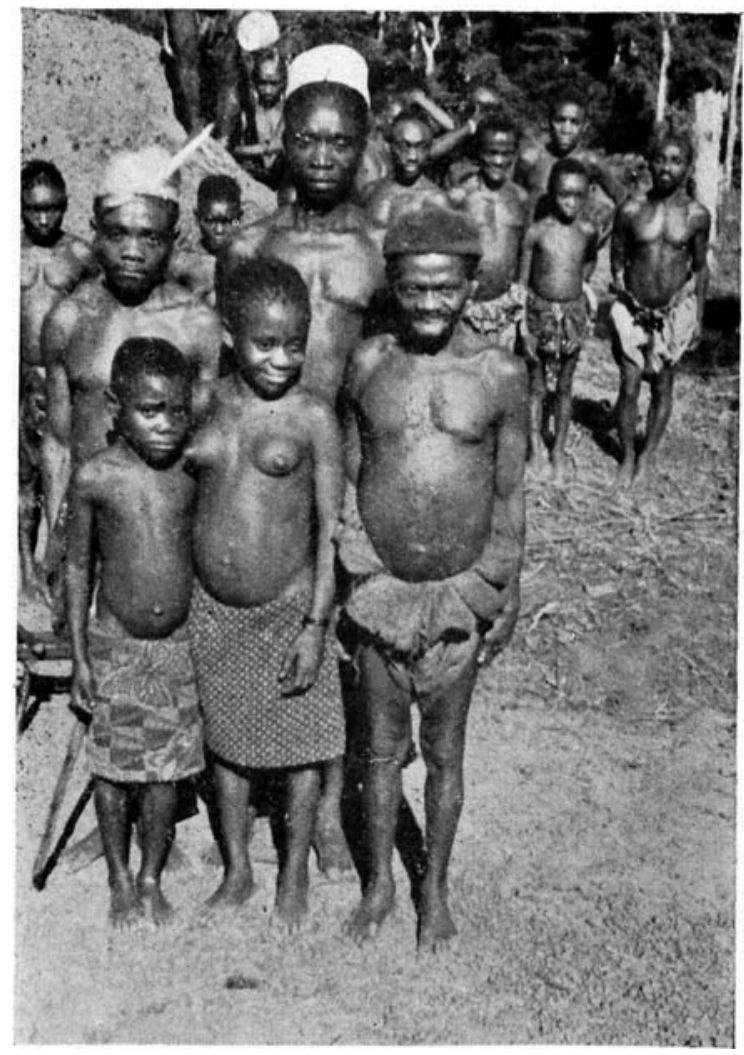

Pigmies of the Ituri forest with an ordinary-sized man for comparison. A recent survey showed an incidence of 6 to 9 per cent. of leprosy, chiefly of a. mild type.

Another puzzling question is why with such a mild type of disease leprosy is so highly endemic. It has been suggested that cases giving negative results on careful and repeated bacteriological examination may yet spread infection through some microscopically unrecognised form of Hansen's bacillus. The general agreement of leprologists is however opposed to this hypothesis. Infectious cases are commoner than might be supposed on superficial inspection. These are the diffuse cases described in $\mathrm{N}$. India and supposed 
by some authorities to be rare elsewhere. However, our inspection of cases at Pawa revealed a fair number of these. The absence of macules and nodules and the hiding of erythema by the darkness of the skin make these cases particularly difficult to recognise at first, until the eye has become accustomed to the skin marking peculiar to this form. Still even when the diffuse lepromatous case is added to the potential disease-spreader the number of dangerous cases is very small.

The danger of the spread of infection in a community is in direct proportion to the number of open cases, and to the opportunities which these open cases have of spreading the disease. In the area uncler discussion the open cases are apparently few; and it must be argued that the opportunities of spreading infection are therefore great.

It has been suggested that the high incidence of leprosy in this region results from dietary deficiency. We found little evidence of the truth of this suggestion. At a centre near Medje, where a complete census of the population was being carried out, we examined some 30 cases of leprosy found. These cases represented all forms of leprosy, and types were roughly in the proportions mentioned above. Leprosy was exceedingly common among the population examined but their physique was excellent and there was little sign of undernourishment or of weakening by accompanying or predisposing diseases. It was noticed, however, that almost all cases were suffering from scabies and septic sores of the skin; that these sores centred chiefly round the gluteal region; and that the majority of slight or early leprous lesions also centred round the same part of the body. It would appear, therefore, that septic sores of this nature are connected either with inoculation of leprous infection or with localisation of the infection once it has been acquired. This skin condition is said to be due to promiscuity with regard to the use of clothes, and especially to the use of unwashable bark cloth.

Another possible facility for the spread of infection may rest with the sexual promiscuity of these people. Not only is polygamy widely practised, wealth being reckoned in the number of wives, but wives are frequently changed. It is not suggested that leprosy is spread as a venereal disease, but promiscuous close contact forms the most favourable condition for transmission.

It must be remembered also that the extreme chronicity of leprosy as found in this area favours high incidence, as the lack of fatality leads to accumulation of cases, whereas in a more acute form the numbers would be more rapidly eliminated by death. Indeed I was informed, though I had not an opportunity of verifying the statement, that in southern provinces of the Congo leprosy 
is more acute, but the number of cases found on survey was smaller.

\section{SUGGiEST IONS FOR CONTROL OF I AEPROSY}

The control of leprosy in the area under consideration is the matter of chief importance. In the Chief's camps all cases are segregated. Of these only about I in Io appears to be a source of danger. Litîle attempt is made, however, to carry out strict isolation of infectious cases. Friends and relations come and go and mix freely with infectious cases. Obviously it would be more efficient to isolate only infectious cases and to isolate them more effectively from the general public. The greatest danger is to the early, slight cases who are segregated along with the most dangerous, no distinction being made. In institutions like that at Li Rangu in the S. Sudan, infectious cases are segregated separately; but I found no attempt at such special segregation in the leper camps I visited in the Belgian Congo. When I suggested that only open cases should be segregated, I was met with the difficulty that the Chiefs and the people could not distinguish between open and closed cases.

Under the present system this cbjection. is of course a very real one. In fact effective leprosy control cannot be carried out without sufficient expert personnel. My suggestion therefore is that in place of, or perhaps in modification of, the present diffuse method more efficient concentrated work be undertaken by expert workers, doctors, ayents sanitaires, etc., thoroughly trained in leprosy work. In such work missions might be asked to co-operate. Missionaries and their native assistants are acquainted with the people and are constantly visiting them. If they had the necessary training with regard to leprosy they might do much towards its control.

Before any such development could take place it would be necessary, however, to appoint a suitable medical expert in leprosy, whose duties would be to study leprosy control and to instruct doctors, agents sanitaires, missionaries and others with a view to their taking part in a campaign against leprosy. Without a wellplanned educational campaign I question whether leprosy control can be carried out with ultimate hope of success.

In conclusion I wish to express my appreciation of the kindness and help received from Prof. Dubois, Dr. de Gotte and others at Pawa, and also from Mr. Harrison and his staff at Ibambi. 